\title{
Binary Systems Within Star Clusters
}

\author{
Ernst Paunzen ${ }^{1,2}$, Christian Stütz ${ }^{2}$, and Bernhard Baumann ${ }^{2}$ \\ ${ }^{1}$ Rozhen National Astronomical Observatory, Institute of Astronomy of the Bulgarian \\ Academy of Sciences, P.O. Box 136, BG-4700 Smolyan, Bulgaria \\ email: ernst.paunzen@univie.ac.at \\ ${ }^{2}$ Institut für Astronomie der Universität Wien, Türkenschanzstrasse 17, \\ A-1180 Wien, Austria
}

\begin{abstract}
WEBDA (http://www.univie.ac.at/webda) is a site devoted to observational data of stellar clusters in the Milky Way and the Small Magellanic Cloud. It is intended to provide a reliable presentation of the available data and knowledge about these objects. The success of WEBDA is documented by its worldwide usage and the related acknowledgements in the literature: more than 650 refereed publications within the last twelve years acknowledged its use. It collects all published data for stars in open clusters that may be useful either to determine membership, or to study the stellar content and properties of the clusters.

The database content includes astrometric data in the form of coordinates, rectangular positions, and proper motions, photometric data in the major systems in which star clusters have been observed, but also spectroscopic data like spectral classification, radial velocities, and rotational velocities. It also contains miscellaneous types of supplementary data like membership probabilities, orbital elements of spectroscopic binaries, and periods for different kinds of variable stars as well as an extensive bibliography. Several powerful tools help to plot, query and extract the data, which can be directly retrieved via http. At the time of writing, about four million individual measurements have been included in the database. The Star Clusters Young \& Old Newsletter (SCYON), a bi-monthly newsletter devoted to star cluster research with about 600 subscribers, is hosted in parallel with the database.

We present the current and upcoming new interface and tools, which are needed to visualize and analyze the increasing amount of data from all-sky surveys, and deeper investigations of binary systems, low mass dwarfs, as well as planet-hosting stars.
\end{abstract}

Keywords. astronomical data bases: miscellaneous, catalogs, open clusters and associations: general, binaries: spectroscopic

\section{New developments within WEBDA}

The current version of the WEBDA open cluster database is comprised basically from files in RDB standard, a clever folder hierarchy and various perl scripts and gnuplot interfaces. At present, a consistent set of tools is developed, to treat all the data in WEBDA homogeneously.

This new suite fuses operations requiring direct access on site with the extraction and charting capabilities provided for the community. Already in coding stage are the following routines:

- Coordinate transformations, automatic scanning for, and refining of, center coordinates.

- The possibility to link and cross check all different datasets for individual clusters.

- Automatic quality and completeness checks to support the previous manually maintained database.

- Charting features we are testing right now include: 
- Displaying coordinates and measurements in charts that allow zooming, filtering objects based on their position, brightness, on the fly.

- Selection of objects within the charts and extracting all available data for these objects.

- Interactive fitting of any displayed, filtered, zoomed sets of data is currently developed.

- Interactive semi-automatic isochrone fitting.

On the technical side, we restrict the suite to freeware or open source tools only. Another requirement we decided on is that there is not anything to be installed on the client side except a web browser capable of interpreting HTML.

We started a statistical analysis of the currently available positional and kinematic data. The analysis is naturally connected with a quality check of the database content and the identification of erroneous entries.

We are confronted with the situation that the availability of B1950 coordinates does not imply that there are also J2000 coordinates for the same object and vice versa. So, we developed several Perl programs which perform the transformation and the calculation of the mean values of stars with available multiple coordinates.

The outcome of this statistical analysis provides us with about 150000 new J2000 coordinates. For about 100000 data points of stars in 446 open clusters, we were able to check the differences of the calculated versus the published J2000 coordinates. From that comparison, we can conclude the proper motions of cluster members and, therefore, the open cluster's proper motion. So, we compiled a new catalogue of 722 cluster proper motions to verify and test our results.

These new coordinates, together with the additional data points in Webda, will help us to create new star charts and e.g. to determine the star cluster's mass.

\section{Binary systems included in WEBDA}

Binary stars are perfect astrophysical laboratories for a number of most important fundamental studies. They represent a significant portion of all stellar systems, and are, therefore, found throughout our Milky Way. Star clusters represent samples of stars of constant age and homogeneous chemical composition, suited for the study of processes linked to stellar structure and evolution, and to fix lines or loci in several most important astrophysical diagrams such as the colour-magnitude diagram, or the Hertzsprung-Russell diagram. Studying binary systems in star clusters is the ideal combination to

- Estimate the binary fraction in correlation with the age and metallicity

- Test the current models of stellar formation and evolution

- Test the current sets of isochrones

- Establish and test the current distances of star clusters with eclipsing binary systems.

Within WEBDA, all published known binary systems within the areas of star clusters are listed in a convenient way. The extraction of all available data for these objects can be easily performed. Currently, there are 1346 spectroscopic and eclipsing binary systems within 134 star clusters available. The whole range of evolutionary stages and metallicities are covered with this sample.

Any feedback to further improve the database would be greatly appreciated.

\section{Acknowledgements}

This work was supported by the financial contributions of the Austrian Agency for International Cooperation in Education and Research (WTZ CZ-10/2010 and HR-14/2010) and the Austrian Research Fund via the project FWF P22691-N16. 